Giovanna Nogueira Pareja ${ }^{1}$ Gabriela Banacu de Melo ${ }^{1}$ Jhosepher Previati de Oliveira ${ }^{2}$ Shajadi Carlos Pardo Kaba ${ }^{2}$ Paulo Henrique Braz-Silva ${ }^{3}$ Camila de Barros Gallo ${ }^{1 *(1)}$
${ }^{1}$ Universidade de São Paulo, School of Dentistry, Stomatology Department - São Paulo - SP - Brasil.

${ }^{2}$ Universidade de Sao Paulo, University Hospital, Oral and Maxillofacial Surgery Department - São Paulo - SP - Brasil.

${ }^{3}$ Universidade de Sao Paulo, School of Dentistry, Pathology Department - São Paulo - SP - Brasil.

Correspondence to:

Camila de Barros Gallo.

E-mail: camilabg@usp.br

Article received on June 20, 2021. Article accepted on September 17, 2021.

DOI: $10.5935 / 2525-5711.20210015$

\section{Oral cancer detection through WhatsApp: the importance of teleconsultation during the COVID-19 pandemic}

\begin{abstract}
:
Introduction: The COVID-19 pandemic has changed the routine of dental care all over the world due to the high risk of contamination by handling body fluids and aerosol production. Biosafety measures were intensified, and dental appointments were restricted to emergencies. In Oral Medicine, the early diagnosis of oral cancer is one of the main urgencies. Objective: The aim of this article is to report a case of oral cancer that was diagnosed during social isolation through a digital messaging platform, in a patient with history of oral potentially malignant disorder. Case Report: The present case report refers to the diagnosis of oral squamous cell carcinoma in an 83-year-old Asian woman with a history of oral potentially malignant disorder. The aforementioned malignant lesion was suspected during a remote consultation using the WhatsApp platform. After the telephone consultation regarding the possible diagnosis, the patient sought care at the referral hospital and underwent clinical examinations, laboratory testing, computed tomography scans and an incisional biopsy to confirm the diagnosis and begin with adequate treatment. Conclusion: The need to mitigate the spread of SARS-CoV-2 prompted the need for and implementation of Teledentistry resources in all fields, including Oral Medicine. Despite being rare, malignant lesions can be diagnosed, as was done in the present report and Oral Medicine plays a crucial role in early diagnosis.
\end{abstract}

Keywords: Remote Consultation; Oral Medicine; Head and Neck Neoplasms; Lichen Planus; Oral 


\section{INTRODUCTION}

On March 11, 2020, WHO has declared the COVID-19 outbreak a global pandemic, caused by the new coronavirus identified as SARS-CoV-2, which changed the entire dynamics of society all over the globe. Dentists are among the professionals who are most at risk of contamination because they handle fluids, such as saliva and blood, and perform procedures that generate aerosols. Thus, dental surgeons, guided by World Health Organization (WHO) guidelines and regional regulatory authorities, began to implement more severe and stringent biosafety standards, to measure patients' body temperature, and to ask questions related to COVID-19 symptoms and recent contact with infected people ${ }^{1,2}$. Furthermore, the mainstream media instructed people to seek dentists only for care of urgent matters in order to maintain social isolation ${ }^{1,3}$.

As regards Oral Medicine, patients with oral lesions that are suspected of being malignant or potentially malignant are considered priority for face-to-face care at this time ${ }^{4}$. There are other urgent demands such as patients with acute and inflammatory lesions of autoimmune or immune-mediated diseases, chronic pain, and oral potentially malignant disorders ${ }^{5,6}$. Many of these conditions specifically affect the elderly population, who are at greater risk of developing severe forms of COVID-19 and were instructed to avoid unnecessary exposure. This scenario intensified the already existing difficulty in the early diagnosis of oral cancer ${ }^{6,7}$.

Whereas the pandemic caused adaptation of and innovation in health care strategies since avoiding interpersonal contact when possible became the new rule. Therefore, alternative forms of awareness, prevention, monitoring, and diagnosis are being made and tested, by using Teledentistry strategies. Video calls, regular phone calls and digital media are the most common means of communication in this context $\mathrm{t}^{6-8}$.

In view of the foregoing, the aim of this study was to report a case of oral cancer that was diagnosed during social isolation through the digital messaging platform WhatsApp, in a patient with history of oral potentially malignant disorder.

\section{CASE REPORT}

In order to assess symptoms and the up to date clinical condition during the social isolation period, the team of the Oral Diagnosis Center placed telephone calls to the patients who had previously been diagnosed with oral potentially malignant disorder or other priority oral diseases.

During one of these calls, the patient, an 83-yearold Asian woman, non-smoker, non-alcoholic, and diagnosed with oral lichen planus, for over 40 years, complained about a burning sensation in the oral mucosa. This patient had been clinically monitored periodically, in at least quarterly intervals, and frequently presented erosive lesions that required treatment based on topical corticosteroids (Figure 1). Furthermore, during the telephone contact, this patient also reported a new asymptomatic lesion in the left buccal mucosa. She was instructed to photograph the area of the complaint and the new lesion, with the help of a family member, and share it via WhatsApp.

In this image, it was possible to observe that the reported asymptomatic lesion was a deep ulcer, with raised, rolled edges and a necrotic center (Figure 2). When asked about the consistency of this tissue, the patient referred to regional thickening. The clinical suspicion was oral squamous cell carcinoma, and the patient was referred for face-to-face care. It was possible to observe that the patient also had candidiasis, which may have been the cause of the primarily reported symptom.

Initially, the family members were strongly opposed to a face-to-face consultation in a hospital environment, as the lesion was asymptomatic and the elderly patient would be exposed to the risk of contamination by SARS-CoV-2, which could result in a fatal outcome. After a new telephone consultation concerning the possibility

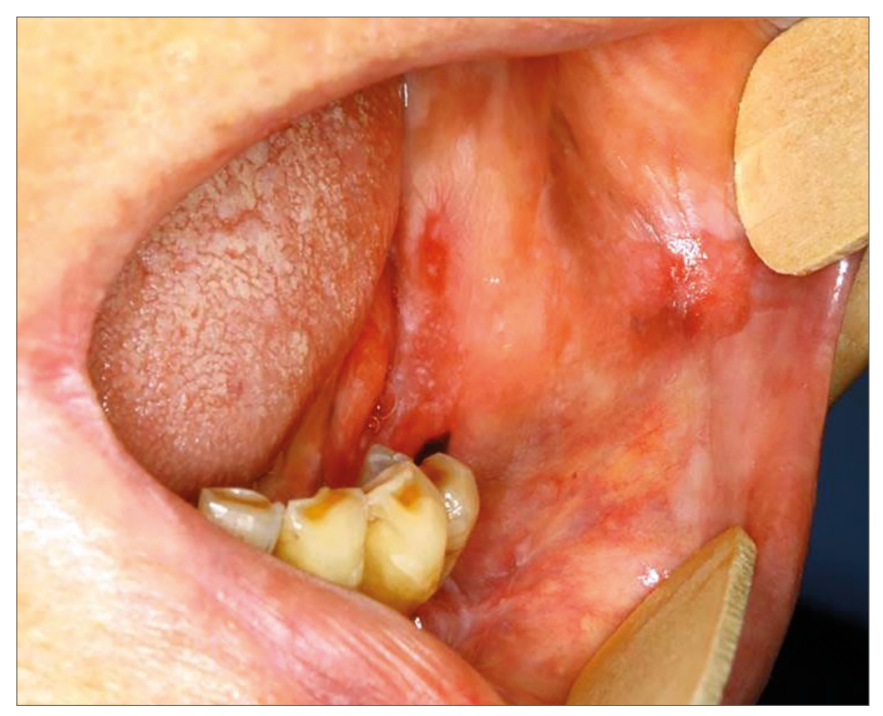

Figure 1. Clinical image of the patient captured in one of the return visits in 2019, showing white papules, striae, and plaques, associated with erythematous and symptomatic atrophic areas in the left cheek mucosa. 


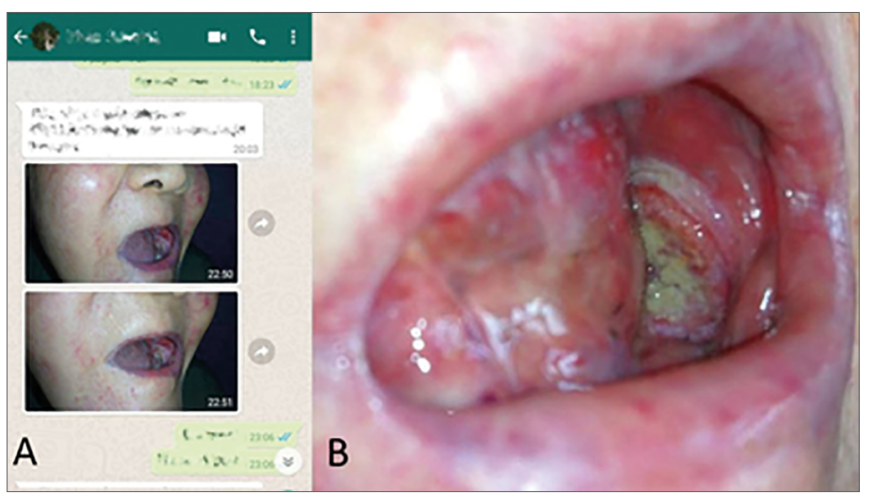

Figure 2. Image of the photo taken by the patient, which was sent by means of the digital platform WhatsApp (A), showing a crateriform ulcerated lesion, with raised edges, necrotic center. According to the patient's report, the lesion was hardened and asymptomatic (B).

of diagnosing an equally serious lesion, they sought care at the referral hospital.

Upon admission to the hospital, the patient underwent a complete clinical examination. The intraoral examination confirmed the presence of a crateriform ulcer in the lowest portion of the left buccal mucosa, measuring approximately $15 \mathrm{~mm}$ in diameter. Additionally, there were white striae and non-raspable plaques associated with erythematous and atrophic areas in the buccal, labial and inferior alveolar ridge mucosa. In the extraoral exam, it was possible to detect a hardened, fixed, and painless submandibular lymph node at level $1 \mathrm{~b}$, measuring approximately 10 millimeters in diameter.

The patient underwent preoperative laboratory testing and computed tomography scans of the head and neck, chest and abdomen for complementary diagnostic and staging purposes. In the image examination, a hypodense, poorly defined formation was observed, which revealed a heterogeneous aspect after contrast with radiopharmaceutical agents. This formation was located on the left lateral wall of the oral cavity, measuring $2.0 \times 1.2 \mathrm{~cm}$ (Figure 3). An incisional biopsy of the ulcerated lesion was performed, which confirmed the diagnosis of oral squamous cell carcinoma, moderately differentiated and invasive. The patient started receiving cancer treatment immediately.

\section{DISCUSSION}

The COVID-19 pandemic changed the way patients seek dental treatment, partly because of the recommendations provided by the WHO guidelines and regional regulatory authorities, in which dental care should be restricted to emergency conditions ${ }^{1-3}$.

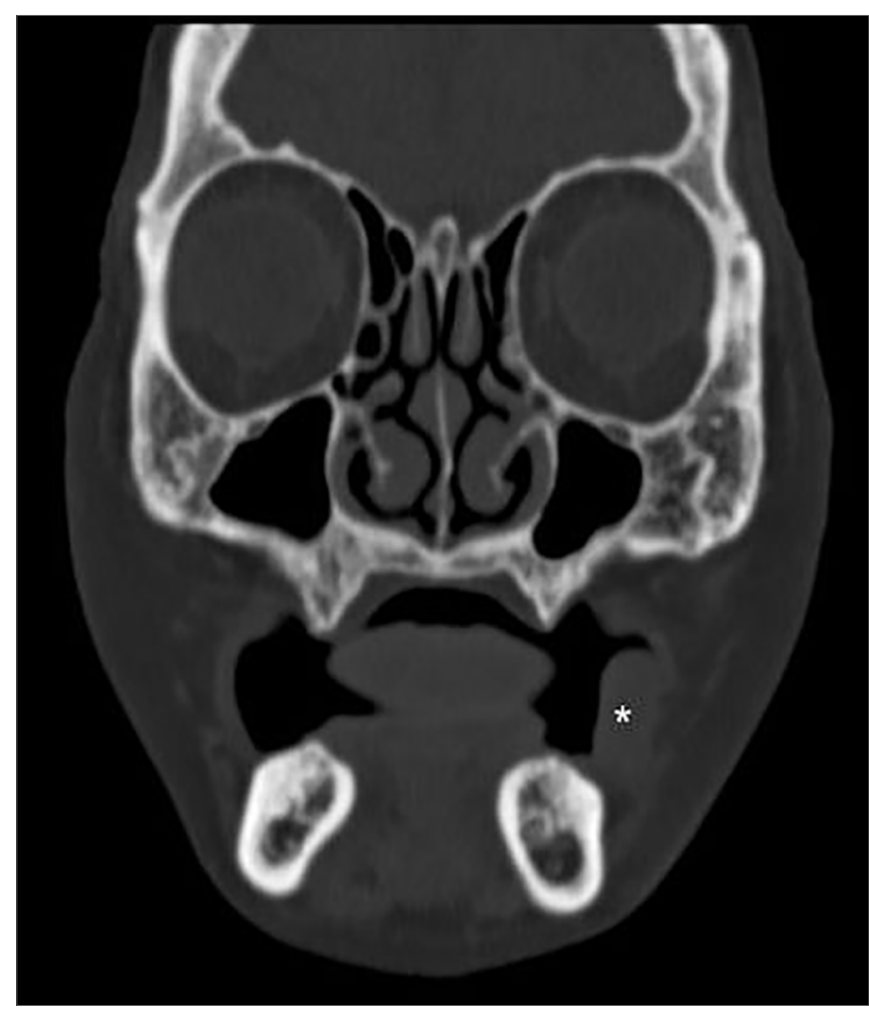

Figure 3. Computed tomography image of a hypodense $\left({ }^{*}\right)$, poorly defined area, on the lateral wall of the left oral cavity, measuring $2.0 \times 1.2 \mathrm{~cm}$.

Tooth pain and orofacial trauma are easily associated with dental emergencies ${ }^{1-3}$. However, some oral mucosal lesions are as important as the cited dental emergencies, including the early diagnosis of oral cancer ${ }^{4}$, monitoring of patients with inflammatory and autoimmune or immune-mediated diseases, chronic pain, and oral potentially malignant disorders ${ }^{5,6}$.

Coupled with the population's lack of knowledge about other dental emergencies, the fear of contamination by the virus that causes severe acute respiratory syndrome has led to many patients who were undergoing cancer therapy to stop going to the hospital for chemotherapy due to their fear of contagion. This has had a negative impact on the prognosis of these patients and this scenario has once again been observed in the present pandemic ${ }^{9}$.

To avoid potentially harmful outcomes, Teledentistry strategies have been widely discussed and tested at this time $e^{6,7}$. The use of the digital platform WhatsApp as a support tool for Telemedicine has been evaluated for some years. Moreover, it demonstrated good results both relative to communication among health professionals, between professionals and their patients, and for learning about health care ${ }^{10}$. 
Specifically in Brazil, Teledentistry was regulated only for consultation between professionals. However, in the face of the pandemic, the Federal Council of Dentistry (CFO) instituted a resolution in June $2020^{11}$, which allowed dental surgeons to engage in teleconsultations to monitor their patients and later on deciding the best time for face-to-face care. This possibility allowed the early diagnosis of the patient reported in this study since her lesion was asymptomatic and had not been recognized by the patient and her family as being worthy of exposure to a face-to-face consultation in order to have it investigated. The early diagnosis of this lesion made it possible for cancer treatment to be implemented immediately, leading towards improved prognosis and better quality of life for the patient ${ }^{7}$

The malignant transformation of oral lichen planus lesions is still a controversial subject that has been widely discussed in the literature. This lesion is classified by WHO as an oral potentially malignant disorder ${ }^{12,13}$. The prevailing systematic reviews on this subject have reported a $1.1 \%$ risk of malignant transformation in patients with oral lichen planus, which is an uncommon event $\mathrm{t}^{12,13}$. This risk is higher in patients who have used or use tobacco (1.98\%), alcohol (2.28\%), were tested positive for hepatitis $\mathrm{C}$ virus (4.46\%) and patients with atrophicerosive lesions $(4.09 \%)$, especially on the tongue ${ }^{13}$. The case reported in this study had no other associated risk factors, except for the atrophic-erosive characteristic of the lesion, diagnosed 40 years ago. One of the possibilities for this would be the epithelial changes present in the lesion of oral lichen planus that resembled oral squamous cell carcinoma, showing a possible genomic instability in the area of liquefaction of the basal layer, which in association with the expression of proliferation and anti-apoptotic biomarkers, can predispose to malignant transformation ${ }^{14}$.

Furthermore, elderly female patients have a greater predisposition for the diagnosis of several malignant lesions, including oral cancer, due to several factors, including their longer life expectancy ${ }^{15}$. Moreover, extensive epidemiological studies have previously demonstrated that lesions in the buccal mucosa are more common in elderly women and specifically in Asian women ${ }^{16}$. However, ethnicity is relevant because of specific harmful habits such as chewing betel quid, which was not observed in this particular patient.

\section{CONCLUSION}

The pandemic reinforced the need to adopt strategies for remote monitoring of patients with oral potentially malignant disorders, including the possibilities offered by Teledentistry. Therefore, although still debatable, monitoring patients diagnosed with oral lichen planus is highly recommended. Despite being rare, malignant lesions can be diagnosed as occurred in the present report, and remote care in Oral Medicine plays a role in early diagnosis.

\section{ACKNOWLEDGMENT}

The authors would like to thank the Programa Unificado de Bolsas de Estudos para Apoio e Formação de Estudantes de Graduação (PUB-Universidade de Sao Paulo) for the scholarship offered to Giovanna Nogueira Pareja and thank Margery Galbraith for the English grammar and style review. The authors have no conflicts of interest or disclosures to declare.

\section{REFERENCES}

1. Spagnuolo G, De Vito D, Rengo S, Tatullo M. COVID-19 outbreak: an overview on dentistry. Int J Environ Res Public Health. 2020 Mar;17(6):2094.

2. Coulthard P. Dentistry and coronavirus (COVID-19) - moral decision-making. Br Dent J. 2020 Apr;228(7):503-5.

3. Peng X, Xu X, Li Y, Cheng L, Zhou X, Ren B. Transmission routes of 2019-nCoV and controls in dental practice. Int J Oral Sci. 2020 Mar;12(1):9.

4. Lopes MA, Santos-Silva AR, Vargas PA, Kowalski LP. Virtual assistance in oral medicine for prioritizing oral cancer diagnosis during the COVID-19 pandemic. Oral Surg Oral Med Oral Pathol Oral Radiol. 2020 Jul;130(1):127-8.

5. Gallo CB, Reis T, Caixeta RAV, Mobile RZ, Costa ALF, Schussel JL, et al. The impact of COVID-19 pandemic in oral medicine and oral pathology practice. J Oral Diag. 2020;5(1):e20200012. DOI: https://doi.org/10.5935/2525-5711.20200012

6. Shanti RM, Stoopler ET, Weinstein GS, Newman JG, Cannady $\mathrm{SB}$, Rajasekaran K, et al. Considerations in the evaluation and management of oral potentially malignant disorders during the COVID-19 pandemic. Head Neck. 2020 Jul;42(7):1497-502.

7. Dolan S. What is the impact of COVID-19 on head and neck squamous cell carcinoma patients? Evid Based Dent. 2020 Jun;21(2):52-3.

8. Machado RA, Souza NL, Oliveira RM, Martelli Júnior H, Bonan PRF. Social media and telemedicine for oral diagnosis and counselling in the COVID-19 era. Oral Oncol. 2020 Jun;105:104685.

9. Chen YM, Perng RP, Chu H, Tsai CM, Whang-Peng J. Impact of severe acute respiratory syndrome on the status of lung cancer chemotherapy patients and a correlation of the signs and symptoms. Lung Cancer. 2004 Jul;45(1):39-43.

10. Giordano V, Koch H, Godoy-Santos A, Belangero WD, Pires RES, Labronici P. WhatsApp messenger as an adjunctive tool for telemedicine: an overview. Interact J Med Res. 2017 Jul;6(2):e11.

11. Conselho Federal de Odontologia (CFO). Resolução CFO226, de 04 de junho de 2020. Dispõe sobre o exercício da Odontologia a distância, mediado por tecnologias, e dá outras providências [Internet]. Brasília (DF): CFO; 2020; [access in 2020 Sep 03]. Available from: http://sistemas.cfo.org.br/ visualizar/atos/RESOLUÇÃO/SEC/ 2020/226 
12. Aghbari SMH, Abushouk AI, Attia A, Elmaraezy A, Menshawy A, Ahmed MS, et al. Malignant transformation of oral lichen planus and oral lichenoid lesions: a meta-analysis of 20095 patient data. Oral Oncol. 2017 May;68:92-102.

13. González-Moles MÁ, Ruiz-Ávila I, González-Ruiz L, Ayén Á, Gil-Montoya JA, Ramos-García P. Malignant transformation risk of oral lichen planus: A systematic review and comprehensive meta-analysis. Oral Oncol. 2019 Sep;96:121-30.
14. Pérez-Sayáns M, Lorenzo-Pouso AI, Chamorro-Petronacci CM, Suárez-Peñaranda JM, Padín-Iruegas E, González-Moles $\mathrm{E}$, et al. Immunoexpression of apoptosis and cell-cycle arrest markers in oral lichen planus. Appl Immunohistochem Mol Morphol. 2020 May/Jun;29(5):374-81. DOI: https://doi. org/10.1097/PAI.0000000000000876

15. Gugic J, Strojan P. Squamous cell carcinoma of the head and neck in the elderly. Rep Pract Oncol Radiother. 2013 Jan;18(1):16-25.

16. Camilon PR, Stokes WA, Fuller CW, Nguyen SA, Lentsch EJ. Does buccal cancer have worse prognosis than other oral cavity cancers? Laryngoscope. 2014 Jun;124(6):1386-91. 